PROCEEDINGS OF THE

AMERICAN MATHEMATICAL SOCIETY

Volume 132, Number 2, Pages 567-577

S 0002-9939(03)07036-9

Article electronically published on September 5, 2003

\title{
ON THE USE OF THE TOPOLOGICAL DEGREE THEORY IN BROKEN ORBITS ANALYSIS
}

\author{
A. V. POKROVSKII AND O. A. RASSKAZOV
}

(Communicated by Michael Handel)

\begin{abstract}
Dynamical systems $f$ in $\mathbb{R}^{d}$ are studied. Let $\boldsymbol{\Omega} \subset \mathbb{R}^{d}$ be a bounded open set. We will be interested in those periodic orbits such that at least one of its points lies inside $\boldsymbol{\Omega}$ and at least one of its points lies outside $\overline{\boldsymbol{\Omega}}$; the orbits with this property are called $\boldsymbol{\Omega}$-broken. Information about the structure of the set of $\boldsymbol{\Omega}$-broken orbits is suggested; results are formulated in terms of topological degree theory.
\end{abstract}

\section{INTRODUCTION}

Consider a continuous mapping $f: \mathbb{R}^{d} \rightarrow \mathbb{R}^{d}$. The $p$ th iteration of $f$ for a positive integer $p$ is denoted by $f^{p}$. A point $x$ is $p$-periodic if $f^{p}(x)=x$. The least positive integer $p=p(x)$ for which $f^{p}(x)=x$ is the minimal period of $x$. For a periodic point $x$ with minimal period $p$ the set of points $\mathcal{O}=\mathcal{O}(x)=\left\{x, f(x), \ldots, f^{p-1}(x)\right\}$ is the orbit of $x$.

Let $\boldsymbol{\Omega} \subset \mathbb{R}^{d}$ be a bounded open set. We are interested in periodic orbits such that at least one of its points lies inside $\boldsymbol{\Omega}$ and at least one of its points lies outside of the closure $\overline{\boldsymbol{\Omega}}$ of $\boldsymbol{\Omega}$. We will call such orbits $\boldsymbol{\Omega}$-broken, or simply broken if there can be no ambiguity. By definition, broken orbits have minimal periods greater than one.

We discuss how topological degree theory can be used in analysis of these broken orbits. If $f: \mathbb{R}^{d} \mapsto \mathbb{R}^{d}$ is a continuous mapping, $\Omega \subset \mathbb{R}^{d}$ is a bounded open set, and $y \in \mathbb{R}^{d}$ does not belong to the image $f(\partial \Omega)$ of the boundary $\partial \boldsymbol{\Omega}$ of $\boldsymbol{\Omega}$, then the symbol $\operatorname{deg}(f, \Omega, y)$ denotes the topological degree 2 of $f$ at $y$ with respect to $\Omega$. If $0 \notin f(\partial \Omega)$, then the number $\gamma(f, \Omega)=\operatorname{deg}(f, \Omega, 0)$ is well defined and it is called the rotation of the vector field $f$ at $\partial \Omega$. The properties of the number $\gamma(f, \Omega)$ are described in detail in [8].

We denote by id the identity mapping: id $(x) \equiv x$. Consider the sequence

$$
\boldsymbol{\alpha}=\boldsymbol{\alpha}(f, \boldsymbol{\Omega})=\left(\alpha_{1}, \alpha_{2}, \alpha_{3}, \ldots\right) \quad \text { where } \quad \alpha_{m}=\gamma\left(\mathrm{id}-f^{m}, \boldsymbol{\Omega}\right) .
$$

A particular element $\alpha_{m}$ of this sequence is well defined if and only if the mapping $f$ has no $m$-periodic points in $\partial \boldsymbol{\Omega}$.

Received by the editors July 28, 2002.

2000 Mathematics Subject Classification. Primary 58C30; Secondary 47H11.

Key words and phrases. Index sequence, topological degree, periodic orbits.

This research was partially supported by the Enterprise Ireland, Grant SC/2000/138. 
We introduce a reduced version of the sequence $\boldsymbol{\alpha}$ which is more suited to the purposes of this article. For each $n \geq 1$, let $J_{n}$ denote the infinite sequence of numbers $\left(j_{1}, j_{2}, \ldots\right)$ where $j_{m}=1$ if $m$ is a multiple of $n$, and $j_{m}=0$ otherwise. For example, $J_{3}=(0,0,1,0,0,1, \ldots)$. We will use the same notation $J_{n}$ for an initial fragment of the sequence $J_{n}$ when there could be no ambiguity. We can define the sequences $\boldsymbol{\alpha}^{(n)}, n=1,2, \ldots$, recurrently by setting $\boldsymbol{\alpha}^{1}=\boldsymbol{\alpha}$ and $\boldsymbol{\alpha}^{(n+1)}=$ $\boldsymbol{\alpha}^{(n)}-\alpha_{n}^{(n)} J_{n}$ for $n=1,2, \ldots$ Here $\alpha_{m}^{(n)}$ denotes the $m$ th element of the sequence $\boldsymbol{\alpha}^{(n)}$. Finally, we introduce the "diagonal" sequence $\boldsymbol{\beta}=\boldsymbol{\beta}(f, \boldsymbol{\Omega})$ defined by $\beta_{1}=0$ and

$$
\beta_{m}=\alpha_{m}^{(m)} \bmod m, \quad m=2,3, \ldots
$$

We note in passing that by construction a particular element $\beta_{m}$ is well defined providing that the element $\alpha_{m}$ is well defined.

For example, for $\boldsymbol{\alpha}=\boldsymbol{\alpha}^{(1)}=(1,3,9,27, \ldots)$, we have

$$
\begin{aligned}
& \boldsymbol{\alpha}^{(2)}=(1,3,9,27, \ldots)-(1,1,1,1, \ldots)=(0,2,8,26, \ldots), \\
& \boldsymbol{\alpha}^{(3)}=(0,2,8,26, \ldots)-(0,2,0,2, \ldots)=(0,0,8,24, \ldots), \\
& \boldsymbol{\alpha}^{(4)}=(0,0,8,24, \ldots)-(0,0,8,0, \ldots)=(0,0,0,24, \ldots) .
\end{aligned}
$$

Thus $\boldsymbol{\beta}=(0,0,2,0, \ldots)$. The elements of the sequence $\boldsymbol{\beta}$ can also be written as

$$
\beta_{m}=\sum \mu(m / p) \gamma\left(\mathrm{id}-f^{p}, \boldsymbol{\Omega}\right) \quad(\bmod m)
$$

where the sum is taken over all divisors $p$ of $m$ and $\mu$ is the Möbius function.

Recall that $\mu(n)$ is defined to be +1 for a positive integer that is a product of an even number of distinct primes, to be -1 if $n$ is a product of an odd number of distinct primes, and to be 0 if $n$ has a multiple prime factor. The conversion of (1.2) to (1.1) is a simple application of the Möbius Inversion Formula (see p. 151, 7]).

Proposition 1.1. Let $f: \mathbb{R}^{d} \rightarrow \mathbb{R}^{d}$ be a map with no $m$-periodic points in $\partial \boldsymbol{\Omega}$, and let $\boldsymbol{\beta}=\boldsymbol{\beta}(f, \boldsymbol{\Omega})$. If $\boldsymbol{\beta}_{m} \neq 0$, then there exists an $\boldsymbol{\Omega}$-broken orbit whose minimal period is a divisor of $m$.

This proposition follows from results of Section 31 of [8]. It is also an immediate corollary of Theorem 2.1.

The sequence $\boldsymbol{\beta}(f, \boldsymbol{\Omega})$ contains plenty of additional information about structure of the set of broken orbits. To explain it is the intention of this paper.

\section{Polynomial systems}

2.1. The result. Let $\mathcal{F}=\mathcal{F}\left(k_{1}, \ldots, k_{d}\right)$ be the set of all polynomial mappings $f: \mathbb{R}^{d} \rightarrow \mathbb{R}^{d}$ given by

$$
(f(x))_{i}=f_{i}(x)
$$

where for each $i$ the component $f_{i}$ has degree no higher than $k_{i}>0$. The list of all coefficients of a particular mapping $f \in \mathcal{F}\left(k_{1}, \ldots, k_{d}\right)$ can be aggregated into a single vector $a(f)$ that belongs to the Euclidean space of the appropriate dimension $K$. Conversely, we can consider the polynomial mapping $f_{a}$ for a given vector $a \in \mathbb{R}^{K}$. The choice of $k_{1}, \ldots, k_{d}$ (and conversely $K$ ) and the method of passing from $\mathcal{F}$ to $\mathbb{R}^{k}$ and back again is fixed for the remainder of this section. 
Recall that a subset $S \subset \mathbb{R}^{K}$ is algebraic if it can be represented as the totality of roots of a polynomial system of equations. Any algebraic set is a closed set with empty interior, has zero Lebesgue measure, and so on.

Theorem 2.1. For each $n$ there exists an algebraic subset $\mathcal{A} \subset \mathbb{R}^{K}$ such that the following holds: if $f=f_{a}$ for some $a \in \mathbb{R}^{K} \backslash \mathcal{A}$ and $m \leq n$ such that $f$ has no $m$-periodic points in $\partial \boldsymbol{\Omega}$ and $\beta_{m}\left(f_{a}, \boldsymbol{\Omega}\right) \neq 0$, then

(a) $f$ has at least one $\boldsymbol{\Omega}$-broken orbit of minimal period $m$ if $m$ or $\beta_{m}$ is odd;

(b) $f$ has an $\boldsymbol{\Omega}$-broken orbit of minimal period $m$ or $m / 2$ if $\beta_{m}$ and $m$ are both even.

Since the set of polynomials is dense in many function spaces, the statements (a) and (b) also hold for generic dynamical systems $f$ with respect to many standard (e.g., $C^{k}$ ) and some less standard topologies. The theorem above is also interesting in its own right, because polynomial approximations of dynamical systems are very common, and many important dynamical systems (for instance, the Hénon mapping) are polynomial mappings.

2.2. Proof of the Theorem. Let $f$ be a differential mapping. A periodic point $y$ of $f$ with minimal period $p$ is $k$-simple if

$$
\operatorname{det}\left(\mathrm{id}-\left(f^{\ell p}\right)^{\prime}(y)\right) \neq 0, \quad \ell=1, \ldots, k .
$$

(We note that this inequality does not follow from $\operatorname{det}\left(\mathrm{id}-\left(f^{k p}\right)^{\prime}(y)\right) \neq 0$; indeed, for $f(x) \equiv-x$ and odd $m$ we have $\operatorname{det}\left(\right.$ id $\left.-\left(f^{m}\right)^{\prime}(0)\right) \neq 0$. However, for even $m$, $\operatorname{det}\left(\right.$ id $\left.\left.-\left(f^{m}\right)^{\prime}(0)\right)=0\right)$. This $k$-simple periodic point $y$ is an isolated fixed point of the mappings $f^{\ell p}, \ell=1, \ldots, k$ and we can consider the numbers

$$
\alpha_{\ell}(y)=\operatorname{ind}\left(y, \text { id }-f^{\ell p}\right)
$$

where ind $(\cdot, \cdot)$ is the Kronecker index [8]. Thus we can consider the finite sequence

$$
\boldsymbol{\alpha}(y)=\left(\alpha_{1}(y), \ldots, \alpha_{k}(y)\right) .
$$

Lemma 2.2. Let $y$ be a $k$-simple point of a differentiable mapping $f$. Then the index sequence (2.2) takes one of four possible forms, namely

$$
\pm(1,1,1,1, \ldots, 1)
$$

or

$$
\pm\left(1,-1,1,-1, \ldots,(-1)^{k-1}\right) .
$$

Moreover, periodic points belonging to the same orbit generate identical sequences.

Proof. This assertion is essentially well known; see [1] or Section 26 of [8]. We will supply a short proof for the reader's convenience.

Denote $B=\left(f^{p}\right)^{\prime}(y)$ and denote by $\sigma_{+}(B)$ (correspondingly $\left.\sigma_{-}(B)\right)$ the number of real eigenvalues of $B$ that are greater than 1 (less than -1 ). By formula (6.8) of [8],

$$
\text { ind }\left(y, \text { id }-f^{\ell p}\right)=(-1)^{\sigma_{+}\left(B^{\ell}\right)}=(-1)^{\sigma_{+}(B)}(-1)^{\sigma_{-}(B)(\ell-1)}, \quad \ell=1, \ldots, k .
$$

Thus the index sequence (2.2) is reduced to the form (2.3) if $\sigma_{-}(B)$ is even, and to the form (2.4) if $\sigma_{-}(B)$ is odd. 
It remains to prove the last part of the lemma. Let $p=p(y)$ be the minimal period of $y$ and let $z \in \mathcal{O}(y)$ with $z \neq y$. So $z=f^{q} y$ for some $1<q<p$. Then ind $\left(y, \mathrm{id}-f^{\ell p}\right)=(-1)^{\sigma_{y}}$ and ind $\left(z\right.$,id $\left.-f^{\ell p}\right)=(-1)^{\sigma_{z}}$ where

$$
\begin{gathered}
\sigma_{y}=\sigma_{+}\left(\prod_{j=0}^{\ell p-1} f^{\prime}\left(f^{j}(y)\right)\right)=\sigma_{+}\left(B_{1} B_{2}\right), \\
\sigma_{z}=\sigma_{+}\left(\prod_{j=0}^{\ell p-1} f^{\prime}\left(f^{j+q}(y)\right)\right)=\sigma_{+}\left(B_{2} B_{1}\right),
\end{gathered}
$$

and

$$
B_{1}=\prod_{j=q}^{\ell p-1} f^{\prime}\left(f^{j}(y)\right), \quad B_{2}=\prod_{j=0}^{q-1} f^{\prime}\left(f^{j}(y)\right) .
$$

Here by $\prod$ we denoted "left-hand side" multiplication, i.e.,

$$
\prod_{j=0}^{q} m_{j}=m_{q} m_{q-1} \ldots m_{1} m_{0} .
$$

However, the sets of eigenvalues together with their multiplicities for the operators $B_{1} B_{2}$ and $B_{2} B_{1}$ coincide and, in particular, $\sigma_{+}\left(B_{1} B_{2}\right)=\sigma_{+}\left(B_{2} B_{1}\right)$. The lemma is thus proved.

The mapping $f$ is said to be $n$-simple if for each $p=1, \ldots, n$ it has only a finite number of periodic points with the minimal period $p$, and each of these points is $\lfloor n / p\rfloor$-simple where $\lfloor\cdot\rfloor$ is the floor function (that is, $\lfloor a\rfloor$ is the largest integer $i$ satisfying $i \leq a)$.

Lemma 2.3. There exists a proper algebraic subset $\mathcal{A}$ of $\mathbb{R}^{K}$ such that for any $a \in \mathbb{R}^{K} \backslash \mathcal{A}$ the polynomial mapping $f=f_{a}$ is $n$-simple.

Proof. First we formulate an auxiliary lemma. Let $\kappa$ be a positive integer and let $F_{\iota}(x ; a), \iota=1, \ldots, \kappa$ be nonzero polynomials, both with respect to $x \in \mathbb{R}^{d}$ and $a \in \mathbb{R}^{K}$.

Lemma 2.4. Let $\mathcal{A}$ be the set of those a for which the simultaneous system

$$
F_{\iota}(x ; a)=0, \quad \iota=1, \ldots, \kappa
$$

has at least one complex solution. Then either $\mathcal{A}=\mathbb{R}^{K}$ or it is contained in a proper algebraic subset of $\mathbb{R}^{K}$.

A short proof of this lemma is relegated to the appendix.

We will come back to the proof of Lemma 2.3. It is sufficient to prove that for a given pair of positive integers $p$ and $\ell$ there exists a proper algebraic subset $\mathcal{A}(\ell, p)$ of $\mathbb{R}^{K}$ such that for any vector $a \in \mathbb{R}^{K} \backslash \mathcal{A}(\ell, p)$ the simultaneous system of equations

$$
f_{a}^{\ell p}(x)-x=0, \quad \operatorname{det}\left(\mathrm{id}-\left(f_{a}^{\ell p}\right)^{\prime}(x)\right)=0
$$

has no complex solutions. By Lemma 2.4 it suffices to construct an open set $U$ of points $a$ for which the system (2.6) is incompatible. Consider the point $a_{*} \in \mathbb{R}^{K}$ that generates the polynomial $f_{a_{*}, i}(\mathbf{x})=x_{i}^{k_{i}}, i=1, \ldots, d$, if $k_{i}>1$, and the 
polynomial $f_{a_{*}, i}(\mathbf{x})=2 x_{i}$, if $k_{i}=1$. The (complex) solutions of the truncated system

$$
f_{a_{*}, i}^{\ell p}(\mathbf{x})-x_{i}=0, \quad i=1, \ldots, d
$$

are exactly the points $y$ for which the component $y_{i}$ is zero if $k_{i}=1$, and, in the case $k_{i}>1, y_{i}$ is either zero or a $\left(k_{i}^{\ell p}-1\right)$-root of unity. For each such solution $y$ the second equation of (2.6) does not hold:

$$
\operatorname{det}\left(\mathrm{id}-\left(f_{a_{*}}^{\ell p}\right)^{\prime}(y)\right)=\prod_{i=1}^{d}\left(1-\left(f_{a_{*}, i}^{\ell p}\right)^{\prime}\left(y_{i}\right)\right) \neq 0 .
$$

Indeed,

$$
1-\left(f_{a_{*}, i}^{\ell p}\right)^{\prime}\left(y_{i}\right)=\left\{\begin{array}{cl}
1-2^{\ell p} & \text { if } k_{i}=1 \\
1 & \text { if } k_{i}>1 \text { and } y_{i}=0 \\
1-k_{i}^{\ell p} & \text { otherwise. }
\end{array}\right.
$$

Therefore (because the degree of each polynomial $f_{a_{*}, i}$ equals $k_{i}$, and roots of a perturbed system cannot "come from infinity"); for a small neighbourhood of $a_{*}$ the corresponding system (2.6) has no complex solutions. The lemma is proved.

Lemma 2.3 is in line with Theorem $\beta$ (on p. 177) of [11]. We note that perturbation of a particular coefficient, say constant perturbation in line with the Sard Lemma, is not enough here. Consider, for example, the system $f(x)=-x$. It has a unique simple fixed point (zero). However, any constant perturbation $f_{u}(x)=f(x)+u$ of this system satisfies $\left(f_{u}\right)^{2}(x) \equiv x$ and thus every point is 2-periodic for any constant perturbation of $f$.

For an $n$-simple mapping $f$ and for an orbit $\mathcal{O}$ of this mapping with minimal period $p \leq n$, we define $b(\mathcal{O})$ to be

$$
b(\mathcal{O})=\sum_{x \in \mathcal{O} \cap \boldsymbol{\Omega}} \text { ind }\left(x, \text { id }-f^{p}\right) .
$$

We denote by $\mathcal{B} O_{p}(f)$ the totality of all $\boldsymbol{\Omega}$-broken orbits of minimal period $p$. Also, we denote by $\mathcal{B} O_{p}^{*}(f)$ the subset of $\mathcal{B} O_{p}(f)$ consisting of those orbits for which the index sequences generated through (2.2) by any point $y$ in the orbit have the form (2.4).

Lemma 2.5. Let $f$ be an $n$-simple mapping and $1 \leq m \leq n$.

(i) If $m$ is odd, then

$$
\beta_{m}=\sum_{\mathcal{O} \in \mathcal{B} O_{m}(f)} b(\mathcal{O})(\bmod m) .
$$

(ii) If $m$ is even, then

$$
\beta_{m}=\sum_{\mathcal{O} \in \mathcal{B} O_{m}(f)} b(\mathcal{O})-2 \sum_{\mathcal{O} \in \mathcal{B} O_{m / 2}^{*}(f)} b(\mathcal{O})(\bmod m) .
$$

Proof. Let us denote by $\boldsymbol{\alpha}(f, \boldsymbol{\Omega}, n)$ the finite sequence of the first $n$ integers of the sequence $\boldsymbol{\alpha}(f, \boldsymbol{\Omega})$. To avoid cumbersome notation we consider only the case when all elements of the sequence $\boldsymbol{\alpha}(f, \boldsymbol{\Omega}, n)$ are well defined.

The sequence $\boldsymbol{\alpha}(f, \boldsymbol{\Omega}, n)$ can be represented as the sum

$$
\boldsymbol{\alpha}(f, \boldsymbol{\Omega}, n)=\sum_{m=1}^{n} \xi_{m} J_{m}
$$


where $\xi_{m}$ are uniquely defined numbers; it suffices to note that the matrix of the corresponding linear system is a triangular one with 1s along the main diagonal.

We will calculate the numbers $\xi_{m}$ in two different ways.

On the one hand, by construction

$$
\boldsymbol{\alpha}(f, \boldsymbol{\Omega}, n)=\sum_{m=1}^{n} \alpha_{m}^{(m)} J_{m}, \quad m=1, \ldots, n .
$$

Indeed, the recurrent procedure given in the introduction is just a description of finding the unknown $\xi_{m}$ by using Gauss's elimination method ([4], p. 23).

On the other hand, by the Kronecker formula (Theorem 4.3, 8] $\alpha_{m}$ is the sum of indices of all fixed points of $f^{m}$ that belong to $\boldsymbol{\Omega}$. According to Lemma 2.2, one can write

$$
\boldsymbol{\alpha}(f, \boldsymbol{\Omega}, n)=\sum_{m=1}^{n}\left(\sum_{\mathcal{O} \in \mathcal{O}_{m}^{(1)}(f)} b(\mathcal{O}) J_{m}+\sum_{\mathcal{O} \in \mathcal{O}_{m}^{(2)}(f)} b(\mathcal{O})\left(J_{m}-2 J_{2 m}\right)\right) .
$$

Here $\mathcal{O}_{p}^{(1)}(f)$ (correspondingly $\mathcal{O}_{p}^{(2)}(f)$ ) denotes the set of orbits of minimal period $p$ whose elements generate sequences (2.3) (the sequences (2.4)).

Comparing (2.11) and (2.12) we obtain

$$
\alpha_{m}^{(m)}=\sum_{\mathcal{O} \in \mathcal{O}_{m}(f)} b(\mathcal{O})
$$

for odd $m$ and

$$
\alpha_{m}^{(m)}=\sum_{\mathcal{O} \in \mathcal{O}_{m}(f)} b(\mathcal{O})-2 \sum_{\mathcal{O} \in \mathcal{O}_{m / 2}^{(2)}(f)} b(\mathcal{O})
$$

for even $m$. Here we have denoted by $\mathcal{O}_{p}(f)$ the totality of all orbits of minimal period $p$.

If a whole orbit of minimal period $m$ is contained in $\boldsymbol{\Omega}$, then its contribution to the right-hand side of $(2.13)$ and $(2.14)$ equals $0(\bmod m)$ due to the last part of Lemma 2.2 In the case of an even $m$, the same fact holds for $m / 2$-periodic orbits belonging to $\Omega$, since their contributions to the right-hand side of (2.14) are doubled. Thus (2.13) and (2.14) are equivalent to (2.8) and (2.9) correspondingly. The lemma is proved.

Now we can give the proof of the theorem. Let $\mathcal{A}$ be an algebraic set that satisfies Lemma 2.3 and let us choose a polynomial mapping $f=f_{a}$ such that $a \notin \mathcal{A}$. The assertion (a) of the theorem follows from the assertion (i) of Lemma 2.5 for the case of odd $m$. It also follows from the assertion (ii) of Lemma 2.5 for the case where $m$ is even and $\beta_{m}$ is odd. The assertion (b) of the theorem follows from the assertion (ii), Lemma 2.5.

\section{BASE PERIOD SETS}

Let $m$ and $b$ be positive integers. A nonempty set $\mathcal{P}$ of positive divisors of $m$ is called the base period set for the pair $(m, b)$ if the greatest common divisor of the set $\mathcal{S}=\{m / p: p \in \mathcal{P}\}$ divides $b$ and the greatest common divisor of any proper subset of $\mathcal{S}$ does not divide $b$. It is convenient also to consider any set $\mathcal{P}$ of positive divisors of $m$ as a base period set for the pair $(m, 0)$. 
For instance, the pair $(12,1)$ has the base sets $\{12\},\{3,4\},\{4,6\}$, whereas the pair $(12,2)$ generates the base period sets $\{12\},\{6\},\{2,3\},\{3,4\}$.

Proposition 3.1. Let $f$ have no $m$-periodic points in $\partial \boldsymbol{\Omega}$ and $\beta_{m} \neq 0$.

(a) If $m$ is odd, then the set of minimal periods for $\boldsymbol{\Omega}$-broken orbits must contain a base period set for the pair $\left(m, \beta_{m}\right)$. (b) If $m$ is even, then there exists a nonnegative integer $\lambda \leq m / 2$ such that the set of minimal periods for $\boldsymbol{\Omega}$-broken orbits contains a base period set for the pair $(m / 2,2 \lambda)$ and a base period set for the pair $(m, m-2 \lambda)$.

Proof. (a) Let $\mathcal{P}(f)$ be the set of those minimal periods for $\boldsymbol{\Omega}$-broken orbits for $f$ that divide $m$. Denote $\mathcal{S}(f)=\{m / p: p \in \mathcal{P}(f)\}$. Consider a sequence of $m$-simple polynomial mappings $g_{i}$ converging to $f$. Such a sequence exists by Lemma 2.3 Then for large $i$ any $\boldsymbol{\Omega}$-broken $m$-periodic orbit $\mathcal{O}$ of $g_{i}$ is close to a $\boldsymbol{\Omega}$-broken orbit of $f$ whose period divides $m$. This implies that

$$
b(\mathcal{O})=\sum_{x \in \mathcal{O} \cap \boldsymbol{\Omega}} \text { ind }\left(x, \text { id }-g_{i}^{p}\right)=\eta s
$$

where $\eta$ is an integer and $s \in \mathcal{S}(f)$. Thus, by assertion (i) of Lemma 2.5, the following equation holds:

$$
\beta_{m}=\sum_{\mathcal{O} \in \mathcal{B} O_{m}\left(g_{i}\right)} b(\mathcal{O})=\sum_{\mathcal{O} \in \mathcal{B} O_{m}\left(g_{i}\right)} \eta(\mathcal{O}) s(\mathcal{O})(\bmod m)
$$

where $s(\mathcal{O}) \in \mathcal{S}(f)$ and $\eta(\mathcal{O})$ are some integer coefficients. This equation has an integer solution with respect to $\eta(\mathcal{O})$ only if the greatest common divisor of the set $\mathcal{S}(f)$ divides $\beta_{m}$. This fact immediately implies that the set $\mathcal{P}(f)$ contains a base period set. The assertion (a) is thus proved.

(b) The proof of the case when $m$ is even resembles the proof given above and so is omitted.

\section{Conclusion and example}

We considered continuous dynamical systems $f$ in $\mathbb{R}^{d}$ and investigated periodic orbits, such that at least one of its points lies inside a given open bounded set $\boldsymbol{\Omega}$ and at least one of its points lies outside $\boldsymbol{\Omega}$. Orbits with this property are called $\boldsymbol{\Omega}$-broken. Interest in $\boldsymbol{\Omega}$-broken orbits arises in many applications, such as models of economy-cycles (where elements of an orbit belonging to the exterior of $\boldsymbol{\Omega}$ represents a "crisis"), models of population dynamics with similar interpretation, power dropout problems of nonlinear optics, etc.

The suggested approach to broken orbit analysis can be useful in many situations. It provides reliable information that is robust with respect to nonsmooth perturbations, including small delays and small hysteresis terms; the corresponding quantitative estimate can also be provided. If the "main part of the model" is "low-dimensional", say not more than 4-dimensional, then it is easy to calculate numerically reasonably long fragments of the corresponding sequences $\boldsymbol{\beta}$ and our method of broken orbits investigation becomes practical for understanding subtle features of the system dynamics. Taking into account the reliability of our approach, it could be combined to a good effect with fast (but often slightly heuristic) algorithms that locate long periodic orbits numerically. In a sequel paper we will consider in detail applications of the methods of this paper to rigorous analysis of unstable periodic orbits in some low-dimensional models in optoelectronics [6], [9]. 
TABlE 1. Experimental results for the Hénon mapping

\begin{tabular}{ccccccccccccccc}
$m$ & 1 & 2 & 3 & 4 & 5 & 6 & 7 & 8 & 9 & 10 & 11 & 12 & 13 & 14 \\
\hline$\alpha_{m}$ & 1 & -1 & 1 & -1 & 1 & -1 & 1 & -1 & 2 & -1 & 0 & 0 & 1 & -1 \\
$\beta_{m}$ & 0 & 0 & 0 & 0 & 0 & 0 & 0 & 0 & 1 & 0 & -1 & 1 & 0 & 0 \\
\hline \hline$m$ & 15 & 16 & 17 & 18 & 19 & 20 & 21 & 22 & 23 & 24 & 25 & 26 & 27 & - \\
\hline$\alpha_{m}$ & 1 & -1 & 0 & -2 & 1 & 1 & 0 & 0 & 0 & 1 & -1 & 0 & -2 & - \\
$\beta_{m}$ & 0 & 0 & -1 & -2 & 0 & 2 & -1 & 2 & -1 & 1 & -2 & 1 & -4 & -
\end{tabular}

TABLE 2. More experimental results for the Hénon mapping

\begin{tabular}{lccccccccccc}
\hline \hline$m$ & $1-7$ & 8 & 9 & 10 & 11 & 12 & 13 & 14 & 15 & 16 & 17 \\
\hline$\beta_{m}$ for $R=0.05$ & 0 & 1 & -2 & 1 & -1 & 1 & -1 & 1 & -1 & 0 & 0 \\
$\beta_{m}$ for $R=0.01$ & 0 & 0 & 0 & 0 & -1 & 1 & -1 & 1 & -2 & 3 & -2 \\
$\beta_{m}$ for $R=0.005$ & 0 & 0 & 0 & 0 & 0 & 0 & 1 & 0 & -1 & 0 & 1 \\
$\beta_{m}$ for $R=0.002$ & 0 & 0 & 0 & 0 & 0 & 0 & 0 & 0 & -1 & 1 & -1 \\
$\beta_{m}$ for $R=0.00175$ & 0 & 0 & 0 & 0 & 0 & 0 & 0 & 0 & 0 & -1 & -1 \\
$\beta_{m}$ for $R=0.0017$ & 0 & 0 & 0 & 0 & 0 & 0 & 0 & 0 & 0 & 0 & 0
\end{tabular}

As an illustrative example, we consider the vectors $\boldsymbol{\beta}(H, \boldsymbol{\Omega})$ for the two-dimensional Hénon mapping [5]. Consider a Hénon mapping $H_{a, b}\left(x^{(1)}, x^{(2)}\right)=\left(1+x^{(2)}-\right.$ $\left.a x^{(1)^{2}}, b x^{(2)}\right)$ with $a=a_{*}=1.3924, \quad b=b_{*}=0.3$. We were interested in the Hénon mapping with these particular values of parameters by the following considerations. For some values of the parameters $a, b$, satisfying $\left|a-a_{*}\right|,\left|b-b_{*}\right|<0.00005$, the fixed point

$$
\left(x_{a, b}^{(1)}, x_{a, b}^{(2)}\right)=\left((b-1) / 2 a-\sqrt{((b-1) / 2 a)^{2}+1 / a}\right)(1, b)
$$

of this mapping generates stable and unstable manifolds that are tangent at some point (i.e., there is a homoclinic tangency). By the classical results of Mora and Viana 10] this implies an abundance of strange attractors for generic diffeomorphisms sufficiently close to $H_{a_{*}, b_{*}}$. Note that the Hénon mapping with the classical parameters was investigated using Zgliczyński's method in [3].

In the first experiment we chose the circle of radius 0.1 centered at the point $(0.64,0.19)$ (the center point was chosen to be reasonably close to the fixed point

$$
\left(x_{*}^{(1)}, x_{*}^{(2)}\right)=\left(\left(b_{*}-1\right) / 2 a_{*}+\sqrt{\left(\left(b_{*}-1\right) / 2 a_{*}\right)^{2}+4}\right)\left(1, b_{*}\right)
$$

of the Hénon mapping $H_{a_{*}, b_{*}}$ ). Due to precision restrictions of the hardware used, we decided to calculate only the first 27 members of the rotation vector. The results are presented in Table 1.

We considered also some other circles with the same center point and different radii, the results of which are presented in Table 2. Since the Hénon mapping is a polynomial, we can interpret results of the experiments using both Theorem 2.1 and Proposition 3.1. For instance, the results show the existence of different broken orbits of the same period located very close to each other. For example, the column for $\beta_{13}$ shows that there exist at least two different broken orbits of length 13 in the open ball of the radius 0.1 centred at $(0.64,0.19)$. We also used a slightly modified 
TABLE 3. Elements $\left(x_{i}^{(1)}, x_{i}^{(2)}\right)$ of a period 15 orbit

\begin{tabular}{lcccccccc}
\hline \hline$i$ & 1 & 2 & 3 & 4 & 5 & 6 & 7 & 8 \\
\hline$x_{i}^{(1)}$ & 0.6303 & 0.6365 & 0.6250 & 0.6471 & 0.6045 & 0.6853 & 0.5273 & 0.8184 \\
$x_{i}^{(2)}$ & 0.1897 & 0.1891 & 0.1910 & 0.1875 & 0.1941 & 0.1813 & 0.2056 & 0.1582 \\
\hline \hline$i$ & 9 & 10 & 11 & 12 & 13 & 14 & 15 & - \\
\hline$x_{i}^{(1)}$ & 0.2256 & 1.1747 & -0.8537 & 0.3376 & 0.5852 & 0.62452 & 0.6325 & - \\
$x_{i}^{(2)}$ & 0.2455 & 0.0677 & 0.3524 & -0.2561 & 0.1013 & 0.1755 & 0.1874 & -
\end{tabular}

program to the find orbit of minimal period 15. Points of this orbit are presented in Table 3.

Technically we used a computer program that calculates the numbers $\gamma($ id $\left.H^{m}, \boldsymbol{\Omega}\right)$ according to the algorithm presented below.

Consider a mapping $f: \mathbb{R}^{2} \mapsto \mathbb{R}^{2}$ and consider a disc $\boldsymbol{\Omega}$ with boundary $\partial \boldsymbol{\Omega}$. The topological degree on $\mathbb{R}^{2}$ coincides with the winding number; thus we can calculate the number $\gamma(\mathrm{id}-f, \boldsymbol{\Omega})$ as follows:

(i) We move from the starting point anti-clockwise until we cross for the first time a coordinate axis. If we do not cross an axis, then the rotation is 0 ; otherwise, we remember which axis we cross.

(ii) We move until we cross the other coordinate axis. Then we observe in which direction we were moving according to the stored values, and add either $+1 / 4$ or $-1 / 4$ to the rotation value. After that, we store new information about the axis we have crossed and continue to move.

(iii) If we encounter the zero vector, the algorithm stops.

The script of the program that we have used to calculate rotation is presented at the website: http://www.ins.ucc.ie/preprints.html. It is based on the algorithm explained above together with adaptive change of the step along $\partial \boldsymbol{\Omega}$. We would like to mention that such an adaptation technique as well as careful "online error control" were essential in our experiments: the Hénon mapping itself looks nice, but its 28th iteration is a polynomial mapping whose degree is of the magnitude $10^{9}$, and the eigenvalues of the linearization of the Hénon mapping at the aforementioned fixed point $\left(x_{*}^{(1)}, x_{*}^{(2)}\right)$ are of the magnitudes $10^{9}$ and $10^{-20}$.

The CPU time to calculate Table 1 was about 1 minute. Taking into account the amount and reliability of the information obtained gives, in our view, credit to the use of topological degree theory in broken orbit investigation.

Acknowledgements. We would like to thank Prof. A. M. Krasnosel'skii, Dr. D. Rachinskii and Dr. S. Wills for discussions and many important suggestions. We also appreciate support of participants of Prof. G. Murphy's Dynamical System Seminar at the University College Cork.

\section{APPENDIX}

5.1. Proof of Lemma 2.4. Let us rewrite the system (2.6) in the coordinate form as

$$
F_{\iota}^{(0)}\left(x_{1}, \ldots, x_{d} ; a\right)=0, \quad \iota=1, \ldots, \kappa_{0}
$$


and denote by $\mathcal{F}^{(0)}$ the set of polynomials $F_{\iota}^{(0)}$. We added the index 0 to initiate the inductive process, where using a modification of the elimination method ([12]), we will construct for each $j=1, \ldots, d$ an algebraic set $\mathcal{A}_{j}$, a nonnegative integer $\kappa_{j}$, and a (possibly empty) set $\mathcal{F}^{(j)}$ of nonzero polynomials

$$
F_{\iota}^{(j)}\left(x_{j+1}, \ldots, x_{d} ; a\right), \quad \iota=1, \ldots, \kappa_{j} .
$$

In particular, polynomials from the last set $\mathcal{F}^{(d)}$ will depend only on $a$. First, we denote by $A^{(j)}$ some square $(d-j) \times(d-j)$ matrices such that all the entries of all the matrices are algebraically independent.

Let $0 \leq j<d$ and suppose that the required integers $\kappa_{j}$ and polynomials $\mathcal{F}^{(j)}$ have been constructed. If $\mathcal{F}^{(j)}=\emptyset$, then define $\mathcal{A}_{j+1}=\emptyset, \mathcal{F}^{(j+1)}=\emptyset, \kappa_{j+1}=0$. Otherwise, for a given $a \in \mathbb{R}^{K}$, the polynomials $F_{\iota}^{(j)}$ have a certain degree $k_{\iota}^{(j)}(a)$ (as polynomials in the $d-j$ variables $x_{j}, \ldots, x_{d}$ ) and set $k_{\iota}^{(j)}=\max _{a} k_{\iota}^{(j)}(a)$. Denote by $\mathcal{A}_{j}^{(1)}$ the set of those coefficients $a$ for which at least one of the polynomials (5.2) has a degree strictly less than $k_{\iota}^{(j)}$. The set $\mathcal{A}_{j}$ is a proper algebraic subset of $\mathbb{R}^{K}$. Define

$$
G_{\iota}^{(j)}\left(x^{(j)} ; a\right)=F_{\iota}^{(j)}\left(A^{(j)} x^{(j)} ; a\right) .
$$

Each polynomial (5.3) has as a polynomial in $x_{j}$ the leading term

$$
g_{\iota}^{(j)}(a) x_{j}^{k_{\iota}^{(j)}}, \quad \iota=1, \ldots, \kappa_{j}
$$

where $g_{\iota}^{(j)}$ is some polynomial of $a$. Denote now by $\mathcal{A}_{j}^{(2)}$ the set of those $a$ that nullify at least one of the polynomials (5.4). We finally denote $\mathcal{A}_{j}=\mathcal{A}_{j}^{(1)} \bigcup \mathcal{A}_{j}^{(2)}$. Below we will use the resultant theory $([12])$. Let $\mathcal{F}^{(j+1)}=\left(F_{1}^{(j+1)}, \ldots, F_{\kappa_{j+1}}^{(j+1)}\right)$ be the set of nonzero elements of the system of $\left(k_{1}^{(j)}, \ldots, k_{\kappa_{j}}^{(j)}\right)$-resultants for the polynomial (5.3) (as polynomials in $x_{j}$ with coefficients in the ring of polynomials of $\left.x_{j+1}, \ldots, x_{d}\right)$.

Recall that $\mathcal{A}$ is the set of those $a$ for which the simultaneous system

$$
F_{\iota}(x ; a)=0, \quad \iota=1, \ldots, \kappa
$$

has at least one complex solution.

Lemma 5.1. (a) The sets $\mathcal{A}_{j}, j=1, \ldots, d$, are algebraic subsets of $\mathbb{R}^{K}$ and do not coincide with $\mathbb{R}^{K}$.

(b) Let $a \notin \bigcup_{j=1}^{d} \mathcal{A}_{j}$. Then $a \in \mathcal{A}$ if and only if a satisfies the simultaneous equations

$$
F(a)=0, \quad F \in \mathcal{F}^{(d)}
$$

Proof. (a) We need only establish that the polynomials (5.4) are nonzero; this follows inductively from the algebraic independence of the elements of the matrices $A^{(j)}$.

(b) For a given $a \notin \mathcal{A}_{j+1}$, compatibility of the simultaneous system

$$
F_{i}^{(j+1)}\left(x_{j+1}, \ldots, x_{d} ; a\right)=0, \quad \iota=1, \ldots, \kappa_{j+1},
$$

is a necessary and sufficient condition for compatibility of the system [12]

$$
G_{\iota}^{(j)}\left(x_{j}, \ldots, x_{d} ; a\right)=0, \quad \iota=1, \ldots, \kappa_{j} .
$$


Also, compatibility of the last system is equivalent to compatibility of the system $F_{\iota}^{(j)}\left(x_{j}, \ldots, x_{d} ; a\right)=0, \quad \iota=1, \ldots, \kappa_{j}$, since the matrices $A^{(j)}$ are invertible. Therefore, the assertion (b) follows by induction.

We now define $\mathcal{A}_{0}$ to be the set of (real) solutions of the system (5.6) and denote $\overline{\mathcal{A}}=\bigcup_{j=0}^{d} \mathcal{A}_{j}$. The set $\mathcal{A}_{0}$ is algebraic and, by the "if" part of assertion (b) of Lemma 5.1 $\mathcal{A} \subset \overline{\mathcal{A}}$. The sets $\mathcal{A}_{\iota}, \iota=1, \ldots, d$, are proper algebraic subsets of $\mathbb{R}^{K}$. If $\mathcal{A}_{0}$ does not coincide with $\mathbb{R}^{K}$, then $\overline{\mathcal{A}}$ is the proper algebraic subset of $\mathbb{R}^{K}$; otherwise $\mathcal{A}$ coincides with $\mathbb{R}^{K}$ by the "only if" part of assertion (b). The lemma is proved.

\section{REFERENCES}

[1] Chow Sh.-N., Mallet-Paret J. and Yorke J.A., A periodic orbit index which is a bifurcation invariant. Geometric dynamics (Rio de Janeiro, 1981), 109-131, Lecture Notes in Math., 1007, Springer-Verlag, Berlin-New York, 1983. MR 85d:58058

[2] Deimling K. Nonlinear functional analysis, Springer-Verlag, 1985. MR 86j:47001

[3] Galias Z. Rigorous numerical studies of the existence of periodic orbits for the Hénon map. SCAN-97 (Lyon). J.UCS 4 (1998), no. 2, 114-124. MR 2000a:37008

[4] Gantmacher F.R. The theory of matrices, Chelsea, New York, 1959. MR 21:6372c

[5] Hénon M., A two-dimensional mapping with a strange attractor, Commun. Math. Phys., 50 (1976), No. 1, 69-77. MR 54:10917

[6] Huyet G., Porta P.A., Hegarty S.P., McInerney J.G. and Holland F., A low-dimensional dynamical system to describe low-frequency fluctuations in semiconductor laser with optical feedback, Optic Communications 180 (2000), 339-344.

[7] Jones G.A. and Jones J.M., Elementary Number Theory, Springer-Verlag, London, 1998. MR 2000b:11002

[8] Krasnosel'skii M.A. and Zabreiko P.P., Geometrical Methods of Nonlinear Analysis, SpringerVerlag, Berlin, 1984. MR 85b:47057

[9] Lang R. and Kobayashi K., IEEE, J. Quantum Electron. 16 (1980) 347.

[10] Mora L. and Viana M. Abundance of strange attractors, Acta Math., 171 (1993), No. 1, pp. 1-71. MR 94k:58089

[11] Shub M., Endomorphisms of compact differentiable manifolds. Amer. J. Math. 91 (1969), 175-199. MR 39:2169

[12] Van der Waerden B.L., Modern Algebra, Vols. I, II. Frederick Ungar Publishing Co., New York, 1949, 1950. MR 10:587b MR 12:236f

Department of Applied Mathematics, National University of Ireland, University College, Cork, Ireland

E-mail address: a.pokrovskii@ucc.ie

Institute for Nonlinear Science, Department of Physics, National University of Ireland, University College, Cork, Ireland

E-mail address: oll@phys.ucc.ie 